\title{
Correlations between various hardness ratios of gamma-ray bursts ${ }^{\star}$
}

\author{
Yi-Ping Qin ${ }^{1,2,3,4}$, Guang-Zhong Xie ${ }^{1,2,4}$, En-Wei Liang ${ }^{1,2,5}$, and Xue-Tang Zheng ${ }^{6}$ \\ 1 Yunnan Observatory, Chinese Academy of Sciences, Kunming, Yunnan 650011, PR China \\ 2 National Astronomical Observatories, Chinese Academy of Sciences \\ 3 Chinese Academy of Science-Peking University joint Beijing Astrophysical Center \\ 4 Yunnan Astrophysics Center \\ 5 Physics Department, Guangxi University, Nanning, Guangxi 530004, PR China \\ 6 Department of Physics, Nanjing University of Science and Technology, Nanjing, Jiangsu 210014, PR China
}

Received 25 February 2000 / Accepted 4 December 2000

\begin{abstract}
We study correlations between various hardness ratios of gamma-ray bursts (GRBs) and investigate if there are any differences between the short- and long-duration classes of the objects in the distributions of the ratios. The results show that most of the hardness ratios are mutually correlated, but that the ratio defined at the lower energy bands is not correlated with those defined at the higher energy bands; the most significant correlations come from those ratios defined with both the lower and higher energy bands. It is also shown that the short-duration bursts tend to have higher values of hardness ratios. We reach these conclusions that the slope of the higher part of the spectrum of most GRBs is independent of that of the lower part; emissions at higher energy bands from the bursts of both short- and long-duration classes are significantly different for different sources, but radiations at lower energy bands are similar; the spectra of the short-duration bursts is harder than that of the long-duration bursts. A possible interpretation for these results involves Doppler boosting in the relativistic beaming model. In addition, the study reveals that hardness ratios of the long-duration class are more mutually correlated than those of the short-duration class; the data of hardness ratios for the long-duration class are much less scattered than those of the short-duration class. It is found that this difference is at least partially due to measurement errors.
\end{abstract}

Key words. gamma rays: bursts - methods: statistical

\section{Introduction}

Gamma-ray bursts (GRBs) are transient astrophysical phenomena in which the emission is confined exclusively to high energies: they are detected at gamma-ray bands and have short lifetimes (from a few milliseconds to several hundred seconds). Since the discovery of such events about thirty years ago (Klebesadel et al. 1973), increasing amounts of data have been obtained (e.g., Fishman et al. 1994; Meegan et al. 1994, 1996, 1998; Paciesas et al. 1997, 1999). Based on the data available thus far, statistical studies have revealed many basic properties of these objects. For example, two separate classes of GRBs were detected: those lasting longer than 2 seconds and those of shorter duration (Dezalay et al. 1992; Hurley 1992; Kouveliotou et al. 1993); furthermore, a correlation be-

Send offprint requests to: Yi-Ping Qin,

e-mail: ypqin@public.km.yn.cn

* This work was subsidized by the Special Funds for Major State Basic Research Projects and by National Natural Science Foundation of China. tween the peak spectral hardness and the peak intensity was discovered (Atteia et al. 1991, 1994, 1997; Belli 1992; Mitrofanov et al. 1992, 1996; Paciesas et al. 1992; Nemiroff et al. 1994; Mallozzi et al. 1995; Dezalay et al. 1997).

More recently, the hardness-duration correlation was confirmed by Fishman (1999) with a large number of bursts observed with BATSE. The hardness ratio is defined as the fluence in channel $3(\sim 100$ to $\sim 300 \mathrm{keV})$ divided by the fluence in channel $2(\sim 50$ to $\sim 100 \mathrm{keV}$ ) (see, e.g., Fishman et al. 1994). This quantity is more likely to present an intrinsic property of the objects than an observational one. In addition to the two channel fluences, there are two other channel fluences available in the BATSE burst catalogs, e.g., channels $1(\sim 20$ to $\sim 50 \mathrm{keV})$ and 4 (>300 keV) (see, e.g., Paciesas et al. 1997). Therefore, one can define more than one hardness ratio. We investigate whether there are any meaningful statistical relations between these different hardness ratios, or between the two classes of the objects in the distributions of these ratios.

In Sect. 2, correlations between various hardness ratios of GRBs will be investigated, while in Sect. 3, differences 
Table 1. The values of $r$ and $p$ for the total sample $(N=1027)$

\begin{tabular}{lllllll}
\hline & & $\log H R_{31}$ & $\log H R_{32}$ & $\log H R_{41}$ & $\log H R_{42}$ & $\log H R_{43}$ \\
\hline $\log H R_{21}$ & $r$ & 0.717 & 0.345 & 0.390 & 0.140 & 0.011 \\
& $p$ & 0.00 & $3.5110^{-21}$ & $4.5910^{-27}$ & $1.8810^{-4}$ & $7.6310^{-1}$ \\
\hline $\log H R_{31}$ & $r$ & & 0.872 & 0.727 & 0.562 & 0.297 \\
& $p$ & 0.00 & 0.00 & 0.00 & $7.2510^{-16}$ \\
\hline $\log H R_{32}$ & $r$ & & 0.728 & 0.687 & 0.393 \\
& $p$ & & 0.00 & 0.00 & $1.5710^{-27}$ \\
\hline $\log H R_{41}$ & $r$ & & & 0.954 & 0.847 \\
& $p$ & & & 0.00 & 0.00 \\
\hline $\log H R_{42}$ & $r$ & & & 0.921 \\
& $p$ & & & & 0.00 \\
\hline
\end{tabular}

Table 2. The values of $r$ and $p$ for the short sample $(N=249)$

\begin{tabular}{lllllll}
\hline & & $\log H R_{31}$ & $\log H R_{32}$ & $\log H R_{41}$ & $\log H R_{42}$ & $\log H R_{43}$ \\
\hline $\log H R_{21}$ & $r$ & 0.610 & -0.148 & 0.173 & -0.207 & -0.210 \\
& $p$ & $1.0210^{-26}$ & $1.9310^{-2}$ & $6.0810^{-3}$ & $1.0410^{-3}$ & $8.6310^{-4}$ \\
\hline $\log H R_{31}$ & $r$ & & 0.620 & 0.663 & 0.406 & 0.165 \\
& $p$ & & $6.9810^{-28}$ & $5.9310^{-33}$ & $2.5810^{-11}$ & $9.0910^{-3}$ \\
\hline $\log H R_{32}$ & $r$ & & 0.672 & 0.729 & 0.403 \\
& $p$ & & $4.7210^{-34}$ & $1.2610^{-42}$ & $3.9110^{-11}$ \\
\hline $\log H R_{41}$ & $r$ & & & 0.906 & 0.806 \\
& $p$ & & & 0.00 & 0.00 \\
\hline $\log H R_{42}$ & $r$ & & & 0.897 \\
& $p$ & & & & 0.00 \\
\hline
\end{tabular}

Table 3. The values of $r$ and $p$ for the long sample $(N=706)$

\begin{tabular}{lllllll}
\hline & & $\log H R_{31}$ & $\log H R_{32}$ & $\log H R_{41}$ & $\log H R_{42}$ & $\log H R_{43}$ \\
\hline $\log H R_{21}$ & $r$ & 0.765 & 0.396 & 0.354 & 0.107 & -0.042 \\
& $p$ & 0.00 & $6.2010^{-28}$ & $2.9810^{-22}$ & $4.5110^{-3}$ & $2.7110^{-1}$ \\
\hline $\log H R_{31}$ & $r$ & & 0.867 & 0.647 & 0.466 & 0.205 \\
& $p$ & 0.00 & 0.00 & $2.2010^{-39}$ & $4.1610^{-8}$ \\
\hline $\log H R_{32}$ & $r$ & & 0.688 & 0.614 & 0.335 \\
& $p$ & & 0.00 & 0.00 & $5.1210^{-20}$ \\
\hline $\log H R_{41}$ & $r$ & & & 0.956 & 0.850 \\
& $p$ & & & 0.00 & 0.00 \\
\hline $\log H R_{42}$ & $r$ & & & & 0.934 \\
& $p$ & & & & 0.00 \\
\hline
\end{tabular}

between the two classes of objects in the distributions of the ratios will be examined. Discussion and conclusions will be presented in Sect. 4 .

\section{Correlations between hardness ratios}

In the 4B catalog (Paciesas et al. 1997), there are 1027 sources with all four channel (1-4) fluences available, providing the "total sample". A hardness ratio $H R_{i j}$ is defined as

$$
H R_{i j} \equiv f_{i} / f_{j}
$$

where $i$ and $j$ denote the channels $i$ and $j$ respectively, and $f_{i}$ and $f_{j}$ represent the fluences of the corresponding channels respectively. The Spearman Rank-Order Correlation coefficients (denoted as $r$ ) and the statistical significance levels (denoted as $p$ ) are presented in Table 1. A small value of $p$ indicates a significant correlation. If $p<0.0001$, then the two quantities are considered to be correlated, otherwise, they are considered uncorrelated. For a given size of sample, if a correlation is significant, the larger value of $r$ corresponds to a more obvious correlation.

From Table 1 we find that almost all hardness ratios are mutually correlated, with the only exceptions of $\log H R_{21}-\log H R_{42}$ and $\log H R_{21}-\log H R_{43}$. The most obvious correlations are $\log H R_{41}-\log H R_{42}$ and $\log H R_{42}-\log H R_{43}$. This suggests that, statistically, the slope of the higher part of the spectrum of most GRBs is independent of the lower part; emissions at higher energy bands from the objects might be significantly different for different sources, while radiations at lower energy bands might be more similar.

\section{Differences between the two classes}

To study the differences between the two classes of GRBs, we examine the data of duration as well as the four channel 

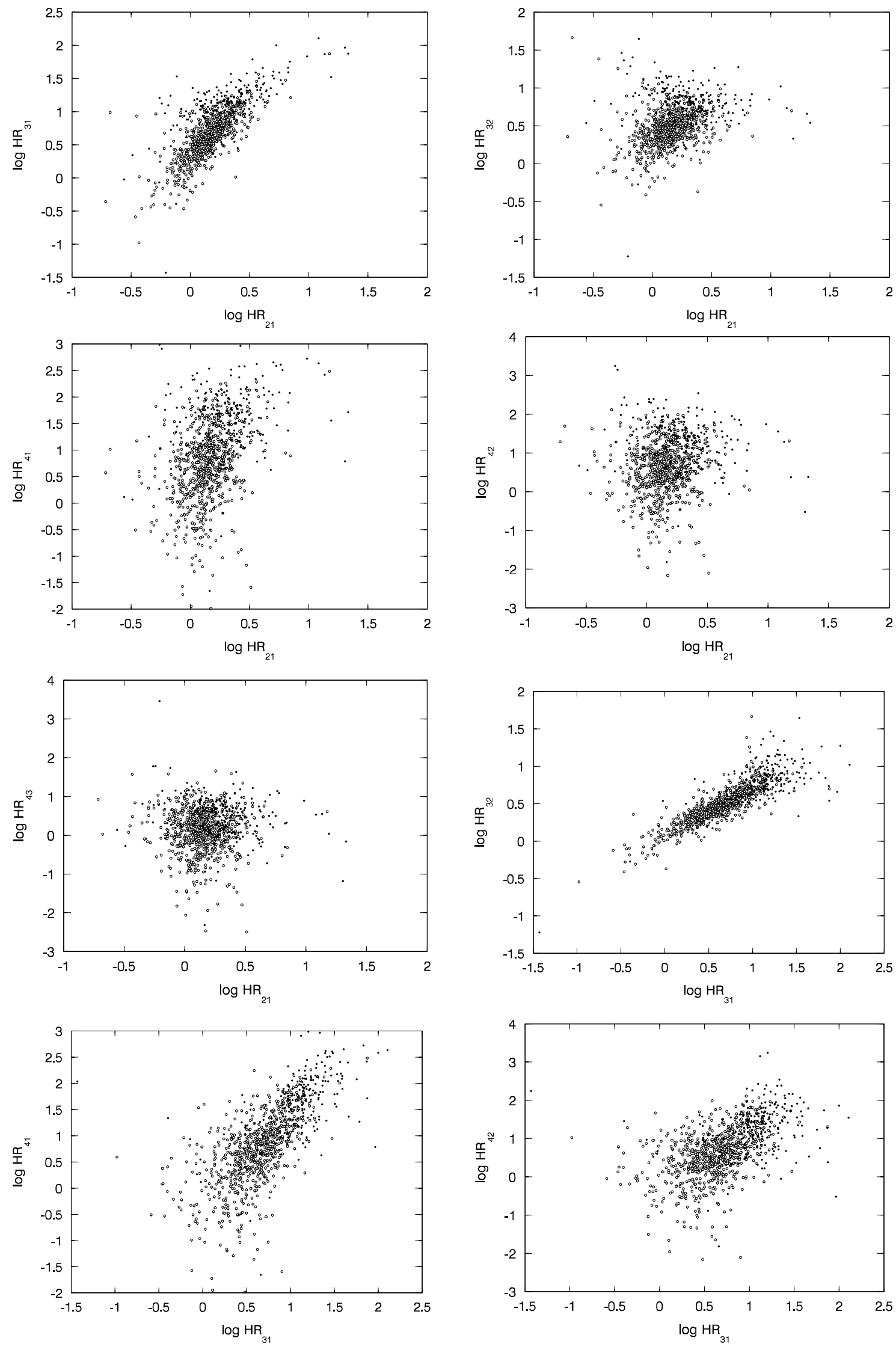

Fig. 1. The plots of $\log H R_{i j}-\log H R_{i^{\prime} j^{\prime}}$ for the short and long samples. The open circle represents a source from the short sample and the filled circle a source from the long sample 

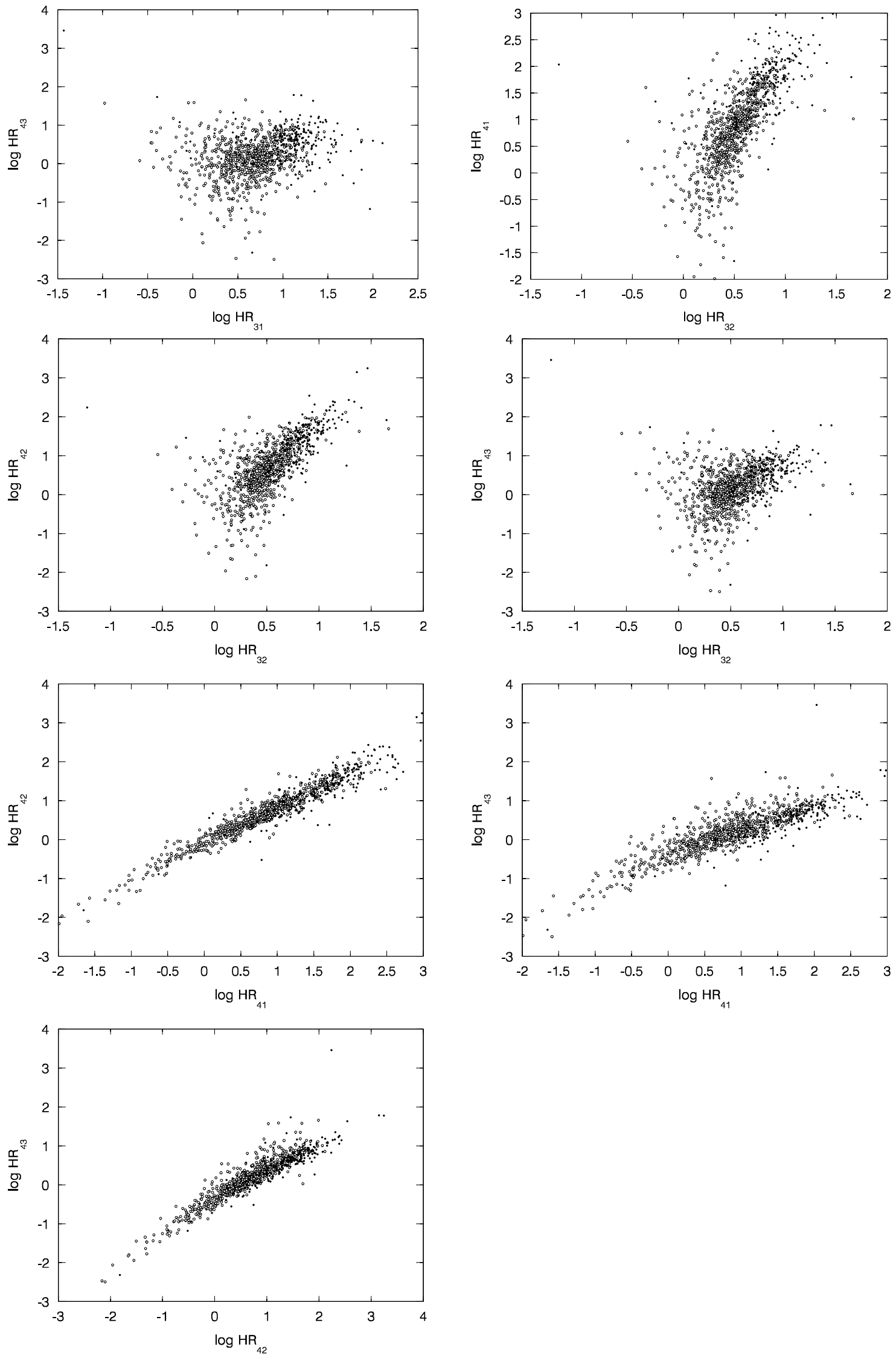

Fig. 1. continued 
fluences available. The bursts are divided into two classes: those sources with $T_{90} \leq 2 \mathrm{~s}$ belong to the short-duration class and those with $T_{90}>2$ s constitute the long-duration class, where $T_{90}$ is the time during which the burst integrated counts increases from $5 \%$ to $95 \%$ of the total counts. We find 249 short-duration sources (called "short sample") and 706 long-duration bursts (called "long sample") from the $4 \mathrm{~B}$ catalog.

The plots of $\log H R_{i j}-\log H R_{i^{\prime} j^{\prime}}$ for the short and long samples are shown in Fig. 1. The Spearman RankOrder Correlation coefficients and the statistical significance levels for the two samples are presented in Tables 2 and 3 respectively.

From Tables 2 and 3 we see that the two classes have the same trend of correlations between hardness ratios shown in the total sample: the majority (10 for the shortduration class and 13 for the long-duration class) of the total 15 pairs of hardness ratios show significant correlations between the corresponding quantities; the ratio defined at the lower energy bands, $\log H R_{21}$, is not correlated with those defined at the higher energy bands, $\log H R_{42}$ and $\log H R_{43}$; the most significant correlations come from those ratios defined with both the lower and higher energy bands, i.e., $\log H R_{41}-\log H R_{42}$ and $\log H R_{42}-\log H R_{43}$. This also suggests that the slope of the higher part of the spectrum of both the short- and long-duration bursts might be independent of the lower part, and statistically, emissions at higher energy bands from the bursts of both classes are significantly different for different sources, while radiations at lower energy bands are more similar.

A difference between the two classes is that hardness ratios of the long-duration class are more mutually correlated than those of the short-duration class (of the total 15 pairs of hardness ratios, $67 \%$ for the short-duration class and $86 \%$ for the long-duration class show correlation between the corresponding hardness ratios). This is consistent with Fig. 1 - the data of hardness ratios for the long-duration class are more concentrated, while that for the short-duration class are more scattered.

Figure 1 also shows different distributions of the two classes. The short-duration bursts tend to occupy the right top part of the panels, showing that, in general, their spectra are harder than that of the long-duration bursts.

\section{Dicussion and conclusions}

In the last two sections, we studied the correlations between various hardness ratios of GRBs for the entire set of $4 \mathrm{~B}$ catalog data (the total sample), the subset containing all short-duration bursts (the short sample), and the subset including all long-duration sources (the long sample). For all of three samples, the results show in common that: (1) the majority (10 for the short-duration class and 13 for both the long-duration class and the entire set of bursts) of the total 15 pairs of hardness ratios show significant correlations between the corresponding quantities; (2) the ratio defined at the lower energy bands, $\log H R_{21}$, is not correlated with those defined at the higher energy bands, $\log H R_{42}$ and $\log H R_{43}$; (3) the most significant correlations come from those ratios defined with both the lower and higher energy bands, i.e., $\log H R_{41}-\log H R_{42}$ and $\log H R_{42}-\log H R_{43}$. Also, we find differences between the two classes: hardness ratios of the long-duration class are more closely correlated than those of the shortduration class.

The major difference between the two classes is consistent with the fact that the data of hardness ratios for the long-duration class are less scattered than those for the short-duration class (Fig. 1). This is most likely due to measurement errors, as the longer bursts tend to have higher fluences and therefore the fluence measurements of the longer bursts will have smaller errors. We calculated the error of hardness ratios with the error transfer formula and found that the errors for shorter bursts are commonly larger than those of longer bursts. For example, the average error of $\log H R_{21}$ for the short sample is 0.26 and for the long sample is 0.05 . The plots of $\log H R_{31}-\log H R_{21}$ with error bars for the short and long samples are shown in Fig. 2. It shows that, at least partially, the difference must be due to measurement errors. (A quantitative study of this issue would involve determining the contribution of errors to the linear correlation analysis. This is beyond the scope of this paper.) Before forming definite conclusions, we must discuss possible impacts on the correlation analysis of the definition of hardness ratios. For a linear correlation analysis of $\log H R_{i j}$ and $\log H R_{i^{\prime} j}$, we are performing a direct comparison between $\log f_{i}-\log f_{j}$ and $\log f_{i^{\prime}}-\log f_{j}$. Note that the two quantities contain an identical variable, $\log f_{j}$. Is the linear correlation analysis between these two quantities in fact the linear correlation analysis between $\log f_{i}$ and $\log f_{i^{\prime}}$ ? The answer is no. For example, if a linear correlation analysis between $\log f_{i}$ and $\log f_{i^{\prime}}$ gives $\log f_{i}=C_{1} \log f_{i^{\prime}}+C_{0}$, one generally cannot expect a result of $\log f_{i}-\log f_{j}=C_{1}\left(\log f_{i^{\prime}}-\log f_{j}\right)+C_{0}$ as well. (We compared the linear correlations between log $f_{1}$ and $\log f_{2}$ and between $\log H R_{41}$ and $\log H R_{42}$, and found that the results are different.) However, if $\log f_{j}$ is negligible compared with $\log f_{i}$ and $\log f_{i^{\prime}}$, then the linear correlation analysis between $\log f_{i}-\log f_{j}$ and $\log f_{i^{\prime}}-\log f_{j}$ must be almost the same as that between $\log f_{i}$ and $\log f_{i^{\prime}}$. On the contrary, compared with $\log f_{j}$, if $\log f_{i}$ and $\log f_{i^{\prime}}$ are negligible, $\log f_{i}-\log f_{j}$ and $\log f_{i^{\prime}}-\log f_{j}$ must be almost identical, and then the linear correlation analysis between these two quantities would give $\log f_{i}-\log f_{j}=D_{1}\left(\log f_{i^{\prime}}-\log f_{j}\right)+D_{0}$, where $D_{1} \simeq 1$ and $D_{0} \simeq 0$, with the correlation coefficient $r \simeq 1$ and the statistical significance level $p \simeq 0$.

We examined whether the most obvious correlation between $\log H R_{41}$ and $\log H R_{42}$ is due to any of these effects. Let us say that $f_{i} / f_{j} \geq 10$ indicates $\log H R_{i j}$ being dominated by $f_{i}$. That is, if $\log H R_{41} \geq 1$ and $\log H R_{42} \geq 1$, then we say $\log H R_{41}$ and $\log H R_{42}$ are dominated by $f_{4}$. On the contrary, if $\log H R_{41} \leq-1$ and $\log H R_{42} \leq-1$, then we say $\log H R_{41}$ and $\log H R_{42}$ 

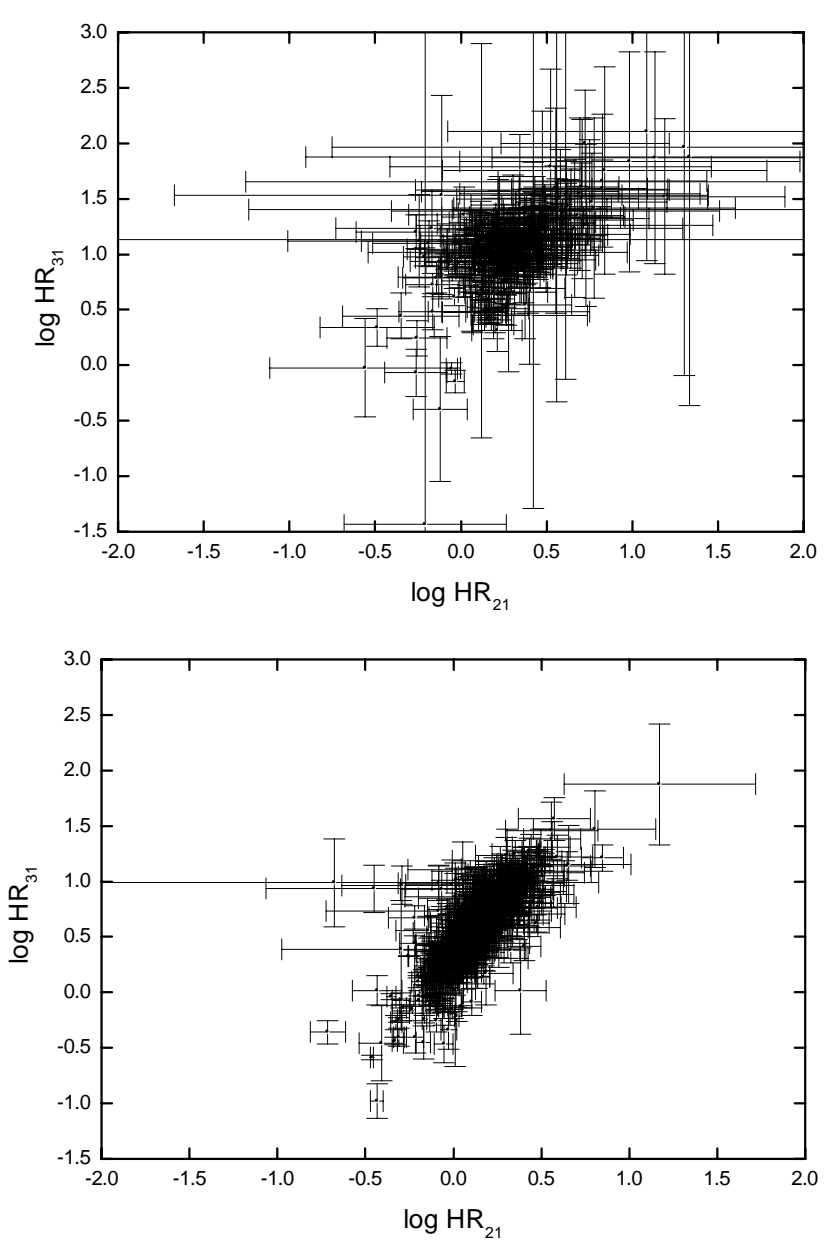

Fig. 2. The plots of $\log H R_{31}-\log H R_{21}$ with error bars for the short (the upper panel) and long (the lower panel) samples

are dominated by $f_{1}$ and $f_{2}$, respectively. In this way, we can redraw the plot of $\log H R_{42}-\log H R_{41}$ using the total sample and adding two boxes - the region of $\log H R_{41} \geq 1$ and $\log H R_{42} \geq 1$ (the right top box), and the area of $\log H R_{41} \leq-1$ and $\log H R_{42} \leq-1$ (the left bottom box) (see Fig. 3). Due to the above effects, we expect that inside the right top box, the two quantities must be correlated, as is the case. Because log $f_{1}$ and $\log f_{2}$ are obviously correlated (the Spearman RankOrder Correlation coefficient is $r=0.976$, with $p=0.0$ ), one can also expect that the two quantities in the left bottom box are also correlated, which is also probably true (see Fig. 3). The area outside these two boxes is not significantly affected by the above effects. Therefore, one would expect a random distribution of data in this area. However, the two quantities are also obviously correlated in this area, and the data inside it lie exclusively along the correlation trend of the two boxes. This suggests that the correlation between $\log H R_{41}$ and $\log H R_{42}$ is intrinsic. Thus, we conclude that the results in common for the three samples suggest, statistically, that the slope of the higher part of the spectrum of most GRBs is independent of the lower part; emissions at higher energy bands from

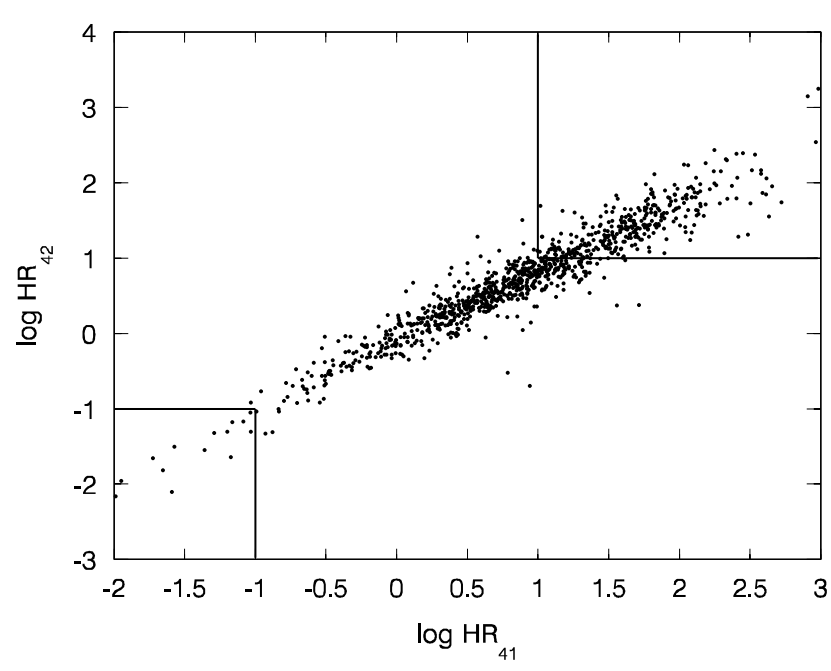

Fig. 3. The plot of $\log H R_{42}-\log H R_{41}$ for the total sample, where the right top box is the region of log $H R_{41} \geq 1$ and $\log H R_{42} \geq 1$ while the left bottom box is the area of $\log H R_{41} \leq-1$ and $\log H R_{42} \leq-1$

the objects are significantly different for different sources, while radiations at lower energy bands are more similar.

Figure 1 also shows different distributions of hardness ratios for the two classes. The short-duration bursts tend to occupy the right top part of the panels, showing that, in general, their spectra are harder than those of the long-duration bursts, confirming the well-known hardnessduration correlation (see, e.g., Fishman 1999). This can be verified by a direct calculation for the two classes. For example, the mean value of $\log H R_{41}$ for the short sample is 1.5 while that for the long sample is 0.67 .

The popular model for GRB spectra is the fourparameter Band GRB function (Band et al. 1993), which has three shape parameters, $\alpha, \beta$, and $E_{\text {peak }} \alpha$ is the power-law index below $E_{\text {peak }}$, while $\beta$ is the index above $E_{\text {peak }} . E_{\text {peak }}$ is typically between 100 and $400 \mathrm{keV}$, i.e., usually in channels 2 and 3 . We suspect that the distribution of the hardness ratios defined with the higher energy band, such as $H R_{41}$, might be due to the distribution of $\beta$, which describes the trend of the spectrum at higher energy bands. We performed a linear correlation analysis between hardness ratios and $\beta$ using the data presented in Band et al. (1993). The results showed no significant correlations between them. For example, there is only a weak positive trend between $\log H R_{41}$ and $\beta$. This might be due to the small size of the sample or the sample selection. We then performed a further analysis of the correlation between $\log H R_{41}$ and $\beta$ using the data presented in Preece et al. (1998), which has a large sample size. Since $\beta$ presented in Preece et al. (1998) is timeresolved with several values for each burst, we used its average value (marked as $\beta_{\text {ave }}$ ). We found that $\log H R_{41}$ and $\beta_{\text {ave }}$ are correlated (the Spearman Rank-Order coefficient is $r=0.59$, with $\left.p=8.6910^{-10}\right)$. The plot of $\beta_{\text {ave }}-\log H R_{41}$ for this sample is shown in Fig. 4 . According to the most successful model proposed so far, 


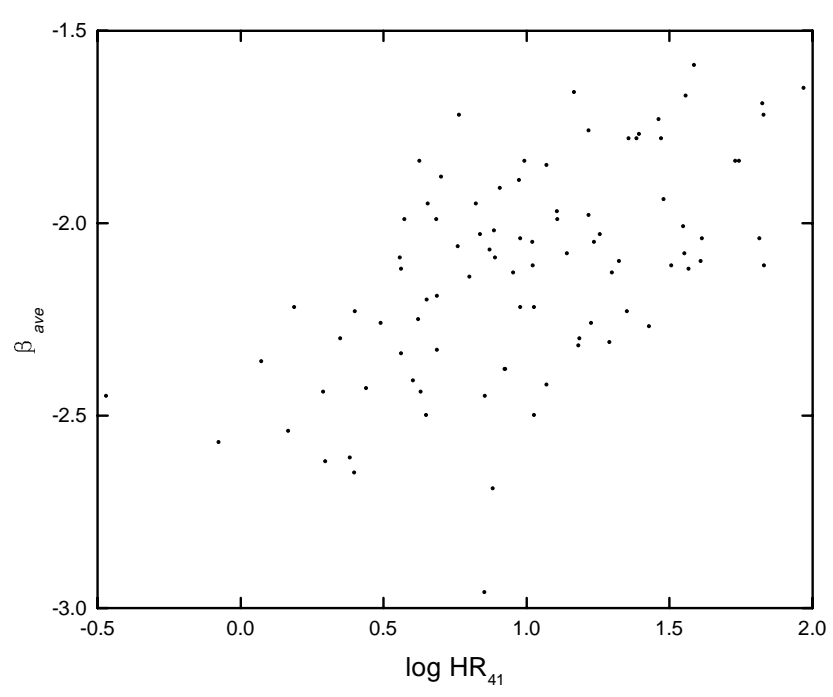

Fig. 4. The plot of $\beta_{\text {ave }}-\log H R_{41}$ for the sample of Preece et al. (1998)

GRBs would result in relativistically expanding fireballs, and the gamma-ray emission would arise after the fireball becomes optically thin, occurring in shocks, either because the ejecta collide with the external medium or because internal shocks occur in a relativistic wind (see Mészáros \& Rees 1997). Several of the features reported in the first GRB, detected over timescales of greater than days at X-ray and optical wavelengths, i.e., GB 970228 (Costa et al. 1997), agreed well with the theoretical expectations of such a model. In the well-known relativistic beaming model used in active galactic nuclei (AGNs) (Rees 1966, 1967), the enhancement of the observed flux for a relativistically moving component is $\delta^{p}$, with $p=3+\alpha$ in the case of a moving sphere and $p=2+\alpha$ in the case of a continuous jet, where $\delta$ is the Doppler factor and $\alpha$ is the spectral index of the component (see, e.g., Ghisellini et al. 1993). Recently, the correlation between polarization and variation for BL Lac objects was found to be applicable to GRBs and a Doppler factor as large as 100 for the bursts was estimated (see Fan et al. 1997, 2001; Cheng et al. 1999). As mentioned above, in contrast to those radiated at lower energy bands, emissions of GRBs at higher energy bands are significantly different for different sources. This leads to the prospect that the Doppler boosting for GRBs might play a more important role at higher energy bands than at lower bands. If so, higher energies must be emitted by the fastest moving ejecta, while lower energies are radiated by the slower moving components in both the isotropic expanding fireball model and the beaming model; or, the range of the spectral index of GRBs at higher energy bands must be significantly wider than that at lower energy bands.
Acknowledgements. This work was supported by the United Laboratory of Optical Astronomy, CAS, and the Natural Science Foundation of Yunnan. We thank the Max-PlanckInstitut fur Radioastronomie, as the main work of revision was carried out by Dr. Yi-Ping Qin when he was a scientific guest there. Thanks are also given to the anonymous referee for the comments that lead to great improvements in this paper.

\section{References}

Atteia, J.-L., et al. 1991, Proc. 22d Int. Cosmic-Ray Conf. (Dublin), 93

Atteia, J.-L., et al. 1994, A\&A, 288, 213

Atteia J.-L., et al. 1997, in AIP Conf. Proc. 384, ed. C. Kouveliotou, M. F. Briggs, \& G. J. Fishman (New York: AIP), 301

Band, D., et al. 1993, ApJ, 413, 281

Belli, B. M. 1992, in AIP Conf. Proc. 265, ed. W. S. Paciesas, \& G. J. Fishman (New York: AIP), 100

Cheng, K. S., et al. 1999 [astro-ph/9910540]

Costa, E., et al. 1997, Nature, 387, 783

Dezalay, J.-P., et al. 1992, in AIP Conf. Proc. 265, ed. W. S. Paciesas, \& G. J. Fishman (New York: AIP), 304

Dezalay, J.-P., et al. 1997, ApJ, 490, L17

Fan, J. H., et al. 1997, A\&A, 327, 947

Fan, J. H., et al. 2001, PASJ, in press

Fishman, G. J. 1999, A\&AS, 138, 395

Fishman, G. J., et al. 1994, ApJS, 92, 229

Ghisellini, G., et al. 1993, ApJ, 407, 65

Hurley, K. C. 1992, in AIP Conf. Proc. 265, ed. W. S. Paciesas, \& G. J. Fishman (New York: AIP), 3

Klebesadel, R., Strong, I., \& Olson, R. 1973, ApJ, 182, L85

Kouveliotou, C., et al. 1993, ApJ, 413, L101

Mallozzi, R. S., et al. 1995, ApJ, 454, 597

Meegan, C. A., et al. 1994, The Second BATSE Burst Catalog, available electronically from the Compton Observatory Science Support Center

Meegan, C. A., et al. 1996, ApJS, 106, 65

Meegan, C. A., et al. 1998, in AIP Conf. Proc. 428, GammaRay Bursts, 4th Huntsville Symp., ed. C. A. Meegan, R. D. Preece, \& T. M. Koshut (New York: AIP), 3

Mészáros, P., \& Rees, M. J. 1997, ApJ, 476, 232

Mitrofanov, L., et al. 1992, in Gamma-ray Bursts, ed. C. Ho, R. I. Epstein, \& E. E. Fenimore (Cambridge: Cambridge Univ. Press), 203

Mitrofanov, L., et al. 1996, ApJ, 459, 570

Nemiroff, R. J., et al. 1994, ApJ, 435, L133

Paciesas, W. S., et al. 1992, in AIP Conf. Proc. 265, ed. W. S. Paciesas, \& G. J. Fishman (New York: AIP), 190

Paciesas, W. S., et al. 1997, The Fourth BATSE Burst Catalog, available electronically at http://cossc.gsfc.nasa.gov/ cossc/batse/4Bcatalog

Paciesas, W. S., et al. 1999, ApJS, 122, 465

Preece, R. D., et al. 1998, ApJ, 496, 849

Rees, M. J. 1966, Nature, 211, 468

Rees, M. J. 1967, MNRAS, 135, 345 